\title{
ALOJAMIENTOS TURISTICOS Y MEDIO AMBIENTE. DIAGNOSTICO AMBIENTAL DE LOS COMPLEJOS DE CABAÑAS EN TANDIL. ARGENTINA.
}

\author{
Autores: \\ Lic. MsC Guillermina Fernández \\ Master en Evaluación de Impacto Ambiental. \\ Universidad Nacional del Centro de la Provincia de Buenos Aires. \\ Lic. Cristina Van Morlegan \\ Licenciada en Diagnóstico y Gestión Ambiental. \\ Lic. Aldo Guzmán Ramos \\ Master en Ecoauditorias y Planificación Empresarial del Medio Ambiente.
}

\section{RESUMEN.}

El presente articulo tiene como objetivo presentar un diagnostico sobre el comportamiento ambiental del sector de alojamientos turísticos de una localidad del sudeste de la Provincia de Buenos Aires en la Republica Argentina.

Se evalúa el sector de cabañas en el área serrana de la localidad de Tandil, por tener en los últimos tiempos un crecimiento superior al resto del sector y localizarse en un área que resulta sensible al impacto del hombre.

El análisis de la situación ambiental de los establecimientos demuestra que existe un claro desconocimiento del entorno y un impacto, que si bien puede ser reducido de forma individual, es importante de manera grupal, por lo cual es necesario considerarlo dentro de una planificación del crecimiento turístico.

Palabras claves: Medio Ambiente, Alojamiento Turistico, Tandil, Crecimiento Turistico

\section{ABSTRACT.}

The present article has as aim present a diagnosis on the environmental behavior of the sector of tourist housings of a locality of the southeast of the Province of Buenos Aires in the Republic Argentina.

The sector of cabins is evaluated in the highland area of the locality Tandil for having in the last times a growth superior to the rest of the sector and to be located in an area that turns out to be sensitive to the impact of the man.

The analysis of the environmental situation of the establishments demonstrates that there exists a clear ignorance of the environment and an impact, which though it can be reduced of individual form, it is important of way grupal, for which it is necessary to consider it inside a planning of the tourist growth. 


\section{INTRODUCCIÓN.}

El turismo se ha convertido en una de las actividades económicas más importantes del mundo. Durante el año 2006, el turismo reportó el 4,5\% de crecimiento en el primer cuatrimestre. Esto se ve reflejado en los ingresos generados, en el número de puestos de trabajo creados, en el movimiento de personas (236 millones de llegadas de turistas internacionales en el mundo), etc., pero en ocasiones ha significado también que los impactos negativos de la actividad, a partir del consumo de recursos y de la producción de desechos, ha ido en aumento.

Este proceso ha desencadenado en muchos destinos una serie de desequilibrios ambientales, que no solo se externalizan en ese espacio geográfico, sino que también se trasladan a otros lugares más lejanos.

Estas consecuencias del crecimiento del turismo deben llevar necesariamente a reflexionar sobre la importancia de lograr armonizar el desarrollo turístico con el ambiente y la sociedad local, siendo necesario que se implemente, en todos los sectores que componen la actividad turística, un modelo de gestión sustentable.

\section{EL TURISMO EN EL MUNDO.}

El surgimiento del turismo, de forma organizada y como actividad económica, podemos situarla en 1814, cuando el inglés Thomas Cook transportó en tren a un grupo de personas de Loughborough a Leicester. Desde ese momento la actividad comenzó a crecer muy lentamente, reducida a los sectores altos de la sociedad. Pero a mediados del siglo XX surgen nuevas transformaciones, muchas de ellas reflejaron mutaciones económicas vinculadas a la revolución industrial (como el aumento del empleo y posteriormente de los ingresos reales, las conquistas laborales, etc.), y generaron un crecimiento del tiempo libre. Esta situación se asoció además a un cambio en la actitud social respecto a la recreación y al ocio, que planteo necesidades de amplios sectores de la sociedad respecto al derecho a disfrutar de este tiempo no laborable. Esto impulsaría entre otras cosas al turismo, y daría paso a un proceso de crecimiento continuo, que actualmente adquiere dimensiones exponenciales en algunos lugares.

Así entonces, y como sostiene la WTTC (World Travels \& Tourism Council) los viajes y el turismo son la mayor actividad del mundo, considerando cualquier indicador económico, como: ingresos brutos, valor añadido, inversiones en capital, puestos de trabajo y contribución impositiva, etc. En 1999 generó, directa o indirectamente, en el conjunto de la economía mundial, el 11\% del Producto Bruto, más de 200 millones de puestos de trabajo (el $8 \%$ del empleo total) y 5,5 millones de nuevos puestos de trabajo por año. Las estimaciones a futuro son que para el 2020 habrá 1,561 millones de llegadas 
de turistas en todo el mundo, esto supondría un gasto de más de 2 billones de dólares, con una tasa media de crecimiento anual de 6.7\%. Particularmente América del Sur se estima conseguirá una tasa de crecimiento del $4.8 \%$ anual del 2000 al 2020, lo que significará un promedio de 43 millones de turistas para el año 2020, siendo su origen principalmente de Europa, Estados Unidos y Canadá.

Todos los datos expuestos sirven para corroborar cuantitativamente la importancia que está teniendo la actividad turística en el mundo (tanto en países desarrollados como subdesarrollados) en estas últimas décadas. También supone reflexionar que a pesar de que en sus comienzos, por el volumen de personas movilizado, el impacto ambiental y social de la actividad no significaba un tema de interés, en la actualidad la expansión extraordinaria del sector hace necesario un cambio de enfoque. Que por lógica debe estar presente aún cuando los volúmenes de capital y de turistas es menor, pero que se acentúa y magnifica cuando se observa el crecimiento y las expectativas del sector.

Así debemos considerar que si bien la actividad turística puede aportar grandes ventajas económicas, principalmente para algunos países subdesarrollados, puede tener también efectos negativos, tanto desde una perspectiva sociocultural como ecológica. Si estos impactos son de cierta magnitud se pone en peligro el desarrollo de la propia actividad turística.

\section{EL SECTOR DE ALOJAMIENTOS TURISTICOS.}

El sector de alojamientos turísticos, en sus diferentes modalidades, juega un papel significativo en el crecimiento del turismo. En términos generales podemos decir que es el «sector que involucra a un conjunto de empresas mercantiles que se dedican de forma profesional y habitual, mediante el cobro de los servicios que prestan, a proporcionar habitación o residencia a las personas que necesitan o desean utilizar esta prestación» (Modulo del Curso de Postgrado en Consultoria Turística. ULPGC. España.).

Este sector conforma una parte importante del sistema turístico, por el movimiento generado, por el rol dentro de los servicios de la oferta y por la contribución en la imagen final del destino. Por tal razón es imprescindible que incorpore mecanismos para lograr una gestión sustentable del servicio, contribuyendo a la calidad de todo el destino. En este caso, como el sector de alojamientos implica cierta infraestructura fija espacialmente, se encuentran comprometidos con el lugar, el deterioro de este implica inexorablemente una pérdida de competitividad no solo del destino sino también para la empresa.

Ahora bien, la realidad indica que estos emprendimientos, de cualquier tamaño, insertos en una economía de libre mercado, suelen basar sus lógicas de acción en una 
racionalidad económica pura, que intenta obtener el máximo beneficio económico, generando externalidades al ambiente y la sociedad. Así surgen numerosos impactos producidos por los alojamientos, uno es el excesivo consumo de agua, del cual la FAO (Organización de las Naciones Unidas para la Agricultura y la Alimentación) estimó que 100 turistas usan en 55 días la misma cantidad de agua que se necesitaría para cultivar arroz para alimentar a 100 personas durante 15 años. Esto implica que es necesario realizar una revisión de estos aspectos y un control en el uso de este recurso y del resto de los utilizados por el sector.

Así, un escenario económico, con mercados cada vez más saturados, consumidores cada vez más exigentes en términos ambientales (sobre todo aquellos parámetros vinculados a calidad de servicios) y una competencia creciente, «obliga»o «estimula» a las empresas turísticas a mejorar sus sistemas de gestión y la calidad de los servicios que ofrecen, y dentro de este cambio se encuadra la gestión ambiental.

Ampliando el marco de análisis se destaca que los servicios ofrecidos por el sector de los alojamientos varían ampliamente. Van desde establecimientos que sólo proveen pernoctación, hasta otros que ofrecen una mezcla de alojamiento, restaurante y servicio de bebidas, así como una serie de actividades adicionales, (directas o indirectas) como celebración de banquetes, actividades de salud y ocio, tiendas, oficina de cambio, salas de conferencias, lavandería, transporte de pasajeros y/o equipaje, garaje, excursiones, actividades de animación, etc.

Todas estas actividades tradicionales o no, implican un proceso que, en un entorno variable, se transforman en las entradas (Input) y salidas (Output) del sistema. $\mathrm{Al}$ respecto, es posible argumentar que «tanto en unas (salidas) como en otras (entradas) encontramos una gran cantidad de productos o sustancias que, en función de su cantidad, composición, origen o forma de elaboración, destino o tratamiento ulterior, etc., inciden de forma diferente sobre el ambiente y sociedad en los que la empresa se inserta. Esta incidencia en el ambiente global y/o local puede afectar, a mediano o largo plazo, la viabilidad de la misma actividad turística» (De Andrés y May. 1995). En realidad es fundamental entender que cualquier unidad considerada, funciona como un subsistema, que a su vez esta influenciada por otro sistema mayor que lo contiene, lo transforma y a su vez es transformado.

Se debe aclarar que si bien las empresas del sector no se caracterizan por causar grandes daños ambientales, sus efectos se componen de toda una serie de pequeñas acciones individuales que pueden producir sinergias negativas sumadas a las de otras empresas (cada una de ellas consumen energía, agua, comida y otros recursos y puede emitir pequeñas cantidades de contaminantes en términos de residuos sólidos, aguas residuales, humo, olores, ruido y algunas sustancias químicas). De esta forma, si se 
considera de forma conjunta el impacto de todas estas pequeñas actividades individuales, así como el elevado número de éstas en ciertos espacios turísticos, el sector de alojamiento tiene un efecto significativo sobre el medio ambiente y los recursos naturales y culturales.

Así, la actividad en su conjunto (mirada fundamental que cada empresario necesita comprender) termina siendo un gran generador de residuos sólidos, un consumidor ineficiente de agua y combustibles fósiles, de grandes cantidades de papel y de una gran cantidad de productos poco respetuosos con el medio ambiente, como por ejemplo plásticos desechables, envases y contenedores no reciclables, productos de limpieza, etc.

Frente a este planteo, en numerosos países del planeta se está incorporando la gestión ambiental en el sector de alojamientos, especialmente en las grandes cadenas hoteleras, que son las primeras que han «sentido la necesidad» por diferentes causas. Los países con mayor desarrollo turístico o mejores políticas sectoriales, como España en Europa, Canadá en América Anglosajona, Costa Rica en América Latina, han avanzado a pasos agigantados en este camino, fundamentalmente porque se incorporó el tema del medio ambiente como factor de competitividad y de diferenciación frente a otros destinos. Un ejemplo de esto es Eco-Index Turismo Sostenible una base de datos de negocios turísticos sostenibles de América y el Caribe que ofrece Rainforest Alliance, como estrategia de promoción de emprendimientos certificados.

Considerando lo expresado, es de fundamental importancia iniciar diagnósticos ambientales de este sector para poder, a partir de allí, implementar mecanismos que reviertan la situación.

\section{DIAGNOSTICO DE LA SITUACION AMBIENTAL DE LAS CABAÑAS EN TANDIL. ARGENTINA.}

\section{Metodología:}

Teniendo en cuenta lo expresado anteriormente, a continuación se presenta el diagnóstico sobre la incorporación de criterios ambientales en la gestión de los complejos de cabañas del Municipio de Tandil. Para esto se toman como eje del diagnóstico, las variables consideradas por uno de los instrumentos de gestión ambiental más relevante en nuestra región, como es el Modelo de Certificación de la Sostenibilidad Turística (CST) del Instituto Costarricense de Turismo. El mismo ha sido retomado por otros mecanismos de certificación regional. Se destaca además el surgimiento desde el 2003 de la red de certificación del Turismo Sostenible a partir de la Conferencia Regional de las Américas sobre certificación de sostenibilidad de las actividades turísticas. Esta red 
es otro elemento clave para los procesos de certificación de la actividad a nivel regional. En general sus principios son compartidos con los de la CST.

A partir de allí se diseño un cuestionario guía que se adaptó a la realidad local y se aplicó a 29 complejos de cabañas en Tandil, que representan el 75\% (considerando los que para el 2005 estaban habilitados y respondieron a los cuestionarios). Los elementos claves que deben ser analizados para el desarrollo de la gestión ambiental en establecimientos hoteleros se basan prioritariamente en cinco áreas: compras, consumo de agua y energía, residuos, cliente externo, a lo que se agregaría la relación con el entorno. Estas áreas son las que identifica representativamente el modelo aplicado.

Para establecer que establecimientos se consideran cabañas, se considero la Ordenanza 8263/01, del Municipio de Tandil, que declara como cabañas a aquellas unidades de vivienda de habitación no permanente, con características arquitectónicas típicas relacionadas con el entorno natural, que mediante acción locativa, se destinen al servicio de alojamiento turístico.

\section{Descripción del espacio geográfico:}

Tandil se localiza en el sudeste de la Provincia de Buenos Aires. Se encuentra a $350 \mathrm{Km}$. de la ciudad de Buenos Aires y la población actual ronda los 108.300 habitantes. El Partido de Tandil está emplazado en la Pampa Húmeda y, más precisamente, en el sistema serrano de Tandilia, que lo atraviesa en sentido NO-SE.

Este sistema orográfico se halla caracterizado por un conjunto de serranías bajas y de formas redondeadas en el Centro y Norte del partido, mientras que cambian a formas de mesas o tabulares hacia el Oeste y Sur. Con respecto al emplazamiento, la ciudad se extiende en una mancha urbana de forma irregular, cuya superficie aproximada es de $50 \mathrm{~km} 2$, y cuyo soporte se caracteriza por ser una encrucijada de valles que descienden desde las Sierras de Tandil hacia la llanura Norte, continuándose hasta la depresión del Río Salado. La ciudad de Tandil está en una zona de interfluvio formada por una lomada al pie y al Norte del Cerro Independencia, y cuya altura es relativamente baja.

Estas características naturales singulares dentro de la región y la mejora en general, del contexto económico en el país, ha permitido que la actividad inmobiliaria, ya sea mediante la promoción, urbanización y construcción, convierta al suelo en un bien altamente rentable y que se empiece a ejercer por ejemplo sobre la zona serrana de Tandil, un incremento notable en la construcción de complejos de cabañas, transformando de manera considerable el elemento de mayor valor y sustento de las actividades turísticas en la zona. 
Este sector esta creciendo a un ritmo mayor que cualquier otro tipo de alojamiento (hotel, motel, posada, etc.) por implicar la posibilidad de una construcción paulatina de los complejos, que van construyendo cabañas a medida que crece el mercado, y además se insertan fácilmente en las zonas serranas.

En términos generales el crecimiento de este sector se ha dado de forma exponencial desde hace menos de una década, acentuándose en el último lustro. Los complejos se ubican en las proximidades de la planta urbana, en el espacio serrano.

\section{Resultados del Diagnóstico Ambiental:}

Las encuestas realizadas revelaron la siguiente información sobre el comportamiento de los complejos de cabañas.

Respecto al consumo de energía, el 100\% utiliza electricidad (de red) y a esto se agrega leña, un $82 \%$, gas envasado un $93 \%$, y solo un $3 \%$ usa energías alternativas, como la energía eólica para el aprovisionamiento de agua. En realidad este consumo, dejando de lado su significado en término de usos de tecnologías no renovables, también supone una pesada carga, sobre todo por los actuales valores del Gas Natural envasado (el que en ocasiones es reemplazado por energía eléctrica a través de radiadores de calor)

Todos los complejos realizan un registro del consumo, pero cuando se consultó particularmente sobre el consumo de cada tipo de energía, solo un 52\% registra el consumo de gas, el $37 \%$ la electricidad y el $31 \%$ el uso de leña.

En cuanto a una escala de uso energético, en primer lugar se encuentra el gas con un $44 \%$, seguido por la leña, $24 \%$, tercero la electricidad, $20 \%$, cuarto gas y electricidad un $6 \%$.

El consumo eléctrico se divide dentro de los complejos entre iluminación y sistemas de frío/calor fundamentalmente.

El consumo de gas se destina en un 55\% a calefacción y se basa en gas natural envasado. El $100 \%$ de la leña usada es destinada para calefaccionar las cabañas, conformando también parte de la imagen promocional de las mismas, ya que en general se encienden las estufas aun en época estival solo por el hecho de que forme parte del paquete ofrecido.

Dos son los ejes sobre los que se identifican ausencia en los criterios de gestión, uno y tal vez el más complejo, y que asocia a una situación estructural es el tipo de 
energía utilizada y el escaso reemplazo por tecnologías alternativas. El otro, y sobre todo prioritario en un proceso de gestión, es la verdadera toma de conciencia y control de una política, no solo de gastos sino de consumo, por áreas, unidades y sectores. Situación que se repite en todas las variables.

Respecto al consumo de agua, punto crucial, solo el 68\% lleva un registro general y un $3 \%$ conoce el gasto por cabaña, pudiendo de esta manera establecer un control mayor.

Consideran el 95\% de los complejos que el sector baño es el que produce el mayor consumo de agua.

Sobre este punto debemos considerar que el $93 \%$ obtiene agua de pozos y solo un $7 \%$ esta conectado a la red de agua corriente. Lo anterior en correlación al tratamiento y disposición de aguas negras, complejiza la situación ambiental y la potencialidad de encontrar acuíferos con riesgos de contaminación orgánica. Por otro lado, y no menos grave es la situación de que en la temporada de verano, estación que cada vez concentra mayor flujo de visitantes, algunos complejos presentan dificultades en el abastecimiento de agua, producto de la saturación en el uso y a su ubicación respecto a la recuperación del acuífero, lo que los obliga a tomar medidas de ahorro forzadas.

Sobre la disposición de las aguas residuales un $31 \%$ tiene un pozo general, un $24 \%$ usa más de un pozo, un $38 \%$ tiene pozo y cámara séptica, esta última permite que se almacenen las natas y sólidos que forman el lodo séptico y una vez realizada la digestión anaeróbica reducen su volumen. Si estos tanques operan de manera adecuada y el mantenimiento es eficaz, el resultado de este proceso es un líquido clarificado y podrá ser vertido en el suelo sin grandes problemas. Solo un 3\% esta conectado a la red cloacal y el resto no declara exactamente la disposición final de sus aguas residuales.

Como se mencionara el hecho de que el 55\% de los complejos de cabañas evacue las aguas residuales a pozos, y sin ningún tratamiento, aumenta las posibilidades de contaminación de las napas freáticas. Esto último se torna aun más peligroso cuando consideramos que el $93 \%$ del consumo de agua de los mismos establecimientos se realiza a través de pozos, sin ningún tipo de tratamiento, y si bien parte es utilizada para tareas de limpieza, riego, etc., una parte también tiene como fin el consumo humano.

Todo esto se produce aunque la Ordenanza 8263/01, expresa que «cada unidad locativa deberá contar con servicios de provisión de agua potable para consumo humano y de desagües cloacales, debiendo evitarse en todos los casos en que no existan redes domiciliarias, que la eliminación de excretas contamine la fuente de aprovisionamiento de agua potable». Los sistemas alternativos de provisión de ambos servicios deberán 
Finalmente, en cuanto a la generación de residuos solo el 31\% lleva un registro de la cantidad de residuos generados.

Este es el panorama del uso de la energía, agua, disposición de residuos y de aguas servidas, a continuación se analizara las medidas implementadas por los complejos para minimizar los impactos.

En primer lugar podemos decir que el $83 \%$ de los complejos implementa mecanismos de ahorro de energía eléctrica, pero únicamente con focos de bajo consumo y un $3 \%$ propende el ahorro de leña, básicamente restringiendo el uso.

Aunque esto último tiene que ver más con el costo de la leña que con una actitud o comportamiento ambientalmente responsable. Por lo tanto si el turista esta dispuesto a pagar más por consumir más leña, el establecimiento (en la mayor parte de los casos) lo proveerá.

Sobre el recurso agua, un 55\% declara considerar el ahorro del mismo revisando las pérdidas periódicamente, un $38 \%$ a través de instalaciones específicas (por ejemplo la gritería monocomando), un $28 \%$ en el riego, implementando parques con vegetación natural, los cuales necesitan mucha menos agua que el césped, un $7 \%$ en las piscinas y un $3 \%$ en el sector de lavandería, terciarizando el sector y en escasa proporción sugiriendo al turista el no recambio diario de la blanquería, de no ser necesario.

En cuanto a los residuos, un 17\% declara tener una política de minimización y reciclado, un $10 \%$ realiza separación de residuos peligrosos (pilas, medicamentos, etc.). Se debe reconocer aquí la necesidad que el sistema de recolección de residuos sólidos urbanos sea el marco para que los establecimientos inicien cierto tipo de estrategias, aunque otras son independientes de la gestión pública.

Respecto a las políticas de compras ambientalmente responsables, un $55 \%$ utiliza productos biodegradables, un 3\% ecológicos, un 3\% productos orgánicos y un $7 \%$ productos reciclados/reutilizados a granel.

Por último, en lo referente al aporte a la educación ambiental del turista, el $48 \%$ declara tener un manual de recomendaciones, expuestos en diferentes unidades dentro del establecimiento y solo un 3\% informa al visitante sobre el patrimonio natural y cultural local. 


\section{CONCLUSIONES.}

El sector de alojamientos turísticos, al igual que el resto de las empresas turísticas, sólo tienen razón de ser en la medida en que el turista se interese por la naturaleza, los atractivos culturales, etc., es decir de elementos que generen la intención de llegar y permanecer en un lugar. Por lo tanto, sería irrisorio e incluso podríamos decir una actitud suicida de parte de los empresarios del ámbito turístico en general y del sector de alojamiento en particular, no contribuir con la preservación y el equilibrio del ambiente y la sociedad local.

Las observaciones reflejan la necesidad de mejorar algunos aspectos de la relación de los complejos de cabañas y el ambiente. Por el momento las actuaciones han sido solo de autorregulación en función de hacer más rentable la inversión. Esto debe ser complementado con otros criterios y con una política y una gestión ambiental integral. Situación que supone una complementariedad con las políticas públicas para la generación de mecanismos de acceso a los servicios, a la información y capacitación, y también un ejercicio de planificación y ordenamiento, regulación y control de las actividades y servicios.

Para concluir, se menciona que estas son algunas de las problemáticas más singulares. La propuesta pretende ser un diagnóstico preliminar y una revisión de la adaptabilidad de la metodología. Se complementa con otros trabajos de clasificación de los complejos de acuerdo a los niveles de compromiso ambiental y con el diseño de una propuesta de transferencia, a partir de un manual de recomendaciones. 


\section{BIBLIOGRAFÍA.}

> Caballero Umpire, R.A. 1995. «Sistema de evaluación del impacto ambiental en proyectos turísticos - Hoteles tipo sol y playa». Tesina presentada para la obtención del Master Internacional de Turismo no publicada. Universidad de Las Palmas de Gran Canaria.

> Céspedes Lorente, José y De Burgos Jiménez, Jerónimo. 1998. «Un análisis del contenido de la gestión ambiental de los establecimientos hoteleros». En Encuentro Medioambiental Almeriense: en busca de soluciones.

$>$ De Andrés, A. y May, C. 1995. Manual ECOTRANS para la mejora de la calidad ambiental en los alojamientos turísticos. Econtrans-España, Madrid.

$>$ Deng, S.L.; Ryan, C. y Moutinho, L. 1992. Canadians hoteliers and their attitudes towards environmental issues. En International Journal of Hospitality Management, 11 (3), pp. 225-237.

> Fernández, Guillermina y Ramos, Aldo G. 2003. Gestión Hotelera. En Gerencia Ambiental. Año 10 No 101. pp. 826-832.

$>$ Fernández, Guillermina y Ramos, Aldo G. 2004. La gestión ambiental en alojamientos turísticos. Un desafío para sustentabilidad y competitividad del sector. En Gestión y Ambiente. Volumen 7. No. 2.

$>$ García Novo, F. 1982. Efectos ecológicos del equipamiento turístico. En Estudios Territoriales 5. pp. 137-144.

$>$ Mathieson, Alister y Wall Geoffrey. 1990. Turismo: repercusiones sociales, económicas y físicas. México. Trillas.

$>$ Organización de Estados Americanos. (OEA) 1997. Sostenibilidad del turismo mediante la gestión de sus recursos naturales y culturales. En XVII Congreso Interamericano de Turismo. San José. Costa Rica.

Recibido: $18 / 04 / 2007$

Aprobado: 04/06/2007

Arbitrado Anónimamente. 
